\title{
Concepciones de padres, profesores y estudiantes, sobre la escuela como escenario de paz
}

\author{
Alexandra Pedraza ${ }^{2}$ \\ Mireya Beatriz Cabrera Yaguana ${ }^{3}$ \\ Aleyda Ángel Florez ${ }^{4}$ \\ Myriam Andrea Leal Velasquez ${ }^{5}$
}

Recibido: 18-08-2018

Aceptado: 03-02-2019

\section{Resumen}

En el marco de una época de reflexión sobre la construcción de paz desde los escenarios educativos, se hace indispensable la participación de la escuela como principal escenario de socialización y formación para la paz. El presente artículo pretende mostrar los resultados obtenidos en una investigación sobre las concepciones

\footnotetext{
1. Artículo de investigación. Producto de la investigación "Concepciones de Padres de Familia, Profesores y Estudiantes de Básica Secundaria, Sobre la Escuela como Escenario de Paz"; grupo de investigación Maestrantes en Asesoría Familiar y Gestión de Programas para la Familia.

2. Universidad de la Sabana. Psicóloga, Especialista en Gerencia Educativa. Profesora de la Maestría en Asesoría familiar y gestión de programas para la familia, Instituto de la Familia, Universidad de La Sabana.

Bogotá - Colombia.

Correo electrónico: Alexandra.pedraza@unisabana.edu.co

ORCID: https://orcid.org/0000-0002-2164-0854

3. Lic. en Ciencias de la Educación especialidad de Psicología Educativa, Universidad Politécnica Saleciana (Cuenca- Ecuador). Mg. en Asesoría Familiar y Gestión de Programas para la Familia, Universidad de La Sabana.

Bogotá - Colombia.

ORCID: https://orcid.org/0000-0002-8955-8427
}

4. Psicóloga, Universidad Católica de Colombia.

Mg. Asesoría Familiar y Gestión de Programas para la Familia, Universidad de La Sabana.

Bogotá - Colombia.

ORCID: https://orcid.org/0000-0003-3169-7229

5. Psicóloga, Universidad de San Buenaventura, Bogotá.

Mg. Asesoría Familiar y Gestión de Programas para la Familia, Universidad de La Sabana.

Bogotá - Colombia.

ORCID: https://orcid.org/0000-0001-9836-2701 
que, respecto a la escuela como escenario de paz, poseen los padres de familia, profesores y estudiantes de básica secundaria de tres instituciones educativas; dos de ellas ubicadas en Colombia, y la otra en Ecuador. Dicha investigación se adelantó desde el enfoque cualitativo, con el objetivo de describir las concepciones de 72 participantes. Los datos fueron recolectados a través de entrevista semiestructurada, validada por expertos; y analizados por medio de codificación y categorización de la información. Se encontró gran afinidad en los resultados de las tres instituciones educativas, en categorías como: paz, familia, convivencia y resolución de conflictos.

Palabras clave: paz, escuela, escenario de paz, convivencia, solución de conflictos.

\title{
Parents, Teachers and Students' Conceptions about School as a Peace Scenario
}

\begin{abstract}
Nowadays, people believe that peace should be build up from the education scenarios, thus, participation of schools for socialization and education about peace is quite important. Accordingly, the main goal of this research is to investigate about parents, teachers and students' conception about schools as a scenario for building up peace. A qualitative approach framework was used in this work. A sample of 72 people from three different middle schools, two located in Colombia and the other one in Ecuador, was evaluated. Data was collected through a semi-structured interview and analyzed by means of codification and categorization. Great affinity was found in the results mainly in categories such as peace, family, coexistence and conflict resolution.
\end{abstract}


Keywords: peace, school, peace scenario, coexistence, solution of conflicts.

\section{Introducción}

Todos los días es posible encontrar a través de los diferentes medios de comunicación, historias en las cuales se registran diversos fenómenos sociales, económicos, políticos y culturales, enmarcados dentro de una convivencia deteriorada, que resalta antivalores como la desigualdad, la inequidad, la injusticia, el irrespeto por los derechos humanos (Pérez, 2013); así como algunas dinámicas de conducta disfuncional que afectan las relaciones interpersonales, y que han trastocado los contextos educativos, y otros ambientes o espacios sociales. Todas las personas, independientemente de su condición socioeconómica, han tenido que vivir directa o indirectamente, los horrores y consecuencias de la guerra, la delincuencia, la desigualdad política y social (Langer, 2016), que al final han venido desvirtuando la posibilidad de vivir en una sociedad en paz; y que ha llevado a otorgarle en gran medida al gobierno, la responsabilidad de alcanzarla. Por ello, es importante entender que la Paz no es simplemente un estado en el que exista armonía en ausencia de conflicto; sino en el cual prevalece la resolución asertiva del conflicto, en pro de una convivencia pacífica; y es en este concepto de Paz, del cual parte la presente investigación.

Autores como Chaux, Lleras y Velásquez (2004), afirman que la convivencia y la paz no hacen referencia a una armonía perfecta, debido a que la multiplicidad de intereses de las personas, generalmente favorecen el roce entre ellos; sin embargo, lo que sí implica una convivencia en paz es "que los conflictos que se presenten sean manejados sin agresión y buscando favorecer los intereses de todas las partes involucradas. Es decir, que las partes 
involucradas logren por medio del diálogo y la negociación, encontrar salidas al conflicto" (p. 19).

Por consiguiente, el conflicto es una de las diversas formas en que pueden interactuar las personas; "en otras palabras, de la misma coexistencia emergen de manera inherente los conflictos en las relaciones humanas que se suscitan en cualquiera de los escenarios de interacción social" (Suárez, 2008, p. 188).

Las personas perciben objetivos e ideas opuestas o contrarias, que incluso interfieren mutuamente en el logro de los objetivos planteados o la expresión de dichas ideas; lo cual ocurre debido a que se ha tenido por mucho tiempo la concepción de que el conflicto es algo negativo, de este modo las actitudes encontradas en un contexto o situación de conflicto, pueden llegar a determinar negativamente el comportamiento y reacción de las personas involucradas en él (Alzate, 2007). Al respecto Ovejero (citado por Suárez, 2008), refiere que "Toda relación social contiene elementos de conflicto, desacuerdos e intereses opuestos. La escuela es una organización y como tal su funcionamiento no puede ser entendido sin considerar la significación del conflicto" (p. 188).

Hoy en día, y a pesar de que hasta hace muy poco, incluso los investigadores sociales tenían la creencia del conflicto como algo negativo, es posible reconocerle como un rasgo social inevitable, inmerso en las relaciones interpersonales. El problema se centra en que:

Todo conflicto puede adoptar un curso constructivo o destructivo y por lo tanto la cuestión no es tanto eliminar o prevenir el conflicto sino saber asumir dichas situaciones conflictivas y enfrentar a ellas con los recursos suficientes para que todos los implicados en dichas situaciones salgamos enriquecidos de ellas. (Alzate, 2007, p. 2)

En otras palabras, Alzate (2007) afirma que en la medida en que sea posible resolver una situación conflictiva, de manera po- 
sitiva, se genera un aprendizaje para quienes intervienen, ello en dos sentidos: el primero se da a nivel de reconocimiento de la existencia de nuevas, diversas y mejores posibilidades de actuar frente a un conflicto que se pueda presentar dentro de procesos de interacción con las demás personas; $y$, el segundo, es el aprendizaje o fortalecimiento de habilidades de resolución de conflictos, lo cual "aumenta la probabilidad de que alcance nuevas soluciones positivas en los conflictos futuros" (p. 2).

Sky (citado por: Fisas, 1998) afirma que "la evolución humana ha ido perdiendo gradualmente el componente cooperativo para favorecer el estrictamente competitivo, base del sistema de dominación" (p. 3). Dicha característica de competitividad en contraposición a la cooperación, ha permeado no solo los escenarios culturales y familiares sino también los escenarios educativos, afectando de manera directa la interacción social en torno a una sana convivencia y marcando una creciente problemática de agresión en las escuelas, donde es posible observar que el mal trato o más conocido fenómeno escolar del Bullying, ha cobrado protagonismo al punto de que los respectivos Ministerios de Educación Nacional de Colombia y Ecuador, al igual que otros en américa latina, han dedicado varios estudios y capítulos al análisis de este fenómeno, buscando soluciones, protocolos y rutas de atención. En este sentido, el Fondo de las Naciones Unidas para la Infancia [UNICEF] y la Facultad Latinoamericana de Ciencias Sociales [FLACSO] (2011), en el documento sobre clima, conflictos y violencia en la escuela, afirman que "para formular políticas educativas que favorezcan una mejora en la convivencia escolar y que permitan trabajar preventivamente, se requiere desarrollar conocimiento sobre la violencia en las escuelas y contar con información rigurosa sobre este fenómeno" (p. 8).

Por ello, el Ministerio de Educación de Ecuador [MEE], UNICEF y World Vision, en el año 2015 adelantaron una investigación sobre el acoso escolar, como forma de violencia entre pares en el sistema educativo en el Ecuador; a través de la cual lograron determinar que las cinco principales formas de violencia escolar, son: los insultos o apodos ofensivos, los rumores o revelación 
de secretos, la sustracción de pertenencias, los golpes, y la agresión por medios electrónicos; además, no se observan diferencias significativas en el sexo de las posibles víctimas; es decir, que se han visto afectados de igual manera por el acoso escolar en alguna de sus formas, tanto hombres como mujeres. Sin embargo, sí se observan diferencias significativas en el sexo de los posibles agresores, encontrando que, por encima del $80 \%$ los agresores han sido hombres. Es así que, toda esta información facilita el abordaje del acoso escolar como una problemática que requiere el fortalecimiento de las estrategias de prevención desde todos los entes coadyuvantes en las dinámicas escolares, donde "las acciones a desarrollar deben lograr una efectiva socialización y conocimiento acerca del acoso escolar, y deben promover cambios en las prácticas y valores para una convivencia pacífica y la resolución no violenta de conflictos" (MEE et al., 2015, p. 72).

Este interés investigativo ha ido creciendo en América Latina, debido al auge que ha tomado la Paz y a la necesidad creciente de generar estrategias para la resolución de conflictos que permitan alcanzarla. Es así que, el Ministerio de Educación de Argentina con el apoyo de la Universidad Nacional de San Martín, inició en 2005 una serie de investigaciones sobre las percepciones que los jóvenes poseen respecto a la violencia en las escuelas, obteniendo así, información tanto teórica como estadística para el fortalecimiento y diseño de políticas públicas con el fin de favorecer la sana convivencia y fortalecerla desde la prevención y la educación (UNICEF y FLACSO, 2011).

En la ciudad de Bogotá, Chaux y Velásquez (2008), realizaron una investigación sobre la prevalencia de la violencia en las escuelas, e hicieron un comparativo con escuelas de otros países como el Salvador, Canadá y Estados Unidos. En dicha investigación se plantearon como variables de estudio algunos tipos de violencia como: agresión física, agresión verbal, exclusión, intimidación o bullying, robos, inseguridad en la escuela, agresión por parte de profesores, uso de armas en peleas o amenazas en la escuela, porte de armas, venta de drogas, y presencia o pertenencia a pandillas. Como resultado de ella, concluyeron que en algunos 
aspectos considerados de agresión leve, no existen diferencias significativas en los reportes hechos en los diferentes países; pero también concluyen, que en aquellas categorías de violencia, que representan un mayor riesgo en las escuelas, y son consideradas presuntos delitos, "como los robos, el porte de armas blancas y la venta de drogas en los colegios de Bogotá, que suceden en frecuencias muy superiores a lo que reportan los estudiantes de otros países" (p. 32).

Los resultados y conclusiones de éstas y otras investigaciones, conllevan a plantear como una necesidad urgente, la prevención de la violencia desde las escuelas como escenarios de construcción de paz; partiendo de la premisa de que desde las instituciones educativas, es posible formar a los estudiantes en aquellas competencias (de conocimiento, cognitivas, emocionales, comunicativas e integradoras), que deben desarrollar para poder relacionarse con sus pares y demás personas, de manera pacífica; así como, generar espacios y ambientes que favorezcan la puesta en práctica de dichas competencias (Chaux et al., 2004; Chaux y Velásquez, 2008; Suárez, 2008).

Dentro del marco legal de esta investigación se considera la Constitución Política de Colombia (1991), que en su artículo 22 enmarca la Paz como "un derecho y un deber de obligatorio cumplimiento" (p. 17). Además, en su artículo 67, delega a la educación la responsabilidad de formar ciudadanos capaces de resolver sus conflictos de manera asertiva y sin necesidad de recurrir a la violencia, basados "en el respeto a los derechos humanos, a la paz y a la democracia" (p. 29). También, está la Constitución de la República del Ecuador (2015), que en su artículo 3, de los deberes primordiales del estado, numeral 8 , afirma que los ecuatorianos tienen "derecho a una cultura de paz, a la seguridad integral y a vivir en una sociedad democrática y libre de corrupción" (p. 17). Igualmente, en su artículo 27 está enunciado que "la educación se centrará en el ser humano y garantizará su desarrollo holístico, en el marco del respeto por los derechos humanos, (...) impulsará la equidad de género, la justicia, la solidaridad y la paz" (p. 27). $Y$, es por esto que, tanto el Ministerio de Educación Nacional de 
Colombia, como el Ministerio de Educación de la República de Ecuador, tienen actualmente priorizada la educación para la paz, los derechos humanos y para la convivencia en el contexto de la escuela, debido a que ésta tiene un gran compromiso en la formación de ciudadanos socialmente responsables, con valores éticos, capaces de ejercer la democracia, respetar los derechos humanos y relacionarse entre sí de manera constructiva y asertiva; con el ánimo de construir una sociedad cada vez mejor para todos (de la calle, Malaver, Gallego, Rodríguez, Flórez, Saldaña, 2014).

Al respecto, Tibbitts (2002) afirma que existen diversos enfoques para abordar la educación en Derechos Humanos, como estrategia de educación para la Paz, el cambio social y el desarrollo humano; pero resalta básicamente tres modelos: el primero de ellos basado en la formación en valores y toma de conciencia, donde propone la integración de dicha formación desde los currículos educativos; el segundo, es un modelo de responsabilidad, donde se propone que cada persona sea responsable respecto de sí y sus ejercicios profesionales, así como de todos aquellos que se encuentran bajo su mando o protección, dando garantía de derechos; y el tercero, es el modelo transformacional, en el cual propone que la persona se empodere y asuma un liderazgo social en la prevención de abusos contra los derechos humanos.

Ahora bien, la responsabilidad de diseñar y poner en marcha políticas y estrategias educativas que conlleven a la configuración de las escuelas como verdaderos escenarios de paz, facilitando la formación de las personas en pro de un cambio social (Tahull, 2016), desde una visión de paz, empoderante y responsable, es de todos los agentes inmersos en el sistema educativo, en sus diferentes niveles de participación. Es así que, tanto el desarrollo personal e integral, como la convivencia pacífica en una sociedad abierta y pluralista, se establece como una necesidad, donde "Estado, sociedad civil, familia y escuela afrontan el reto de la formación para la convivencia no sólo como una cuestión de hecho, sino como un compromiso de voluntades hacia lo que es valioso en la educación" (Reboul, 1972; Tedesco, 1995; Morín, 2000; Touriñán, 2004a; citados por: Touriñán, 2007; p. 263). 
Es entonces, fundamental el papel que cumple la educación en la formación en competencias ciudadanas, que le permiten al ser humano convertirse en un ciudadano, enfrentando los diversos retos que se le presentan; como afirman Chaux et al. (2004):

Por un lado, está el reto de convivir pacífica y constructivamente con otros que frecuentemente tienen intereses que riñen con los nuestros. En segundo lugar, está el reto de construir colectivamente acuerdos y consensos sobre normas y decisiones que nos rigen a todos y que deben favorecer el bien común. Y, en tercer lugar, el ejercicio de la ciudadanía implica el reto de construir sociedad a partir de la diferencia, es decir, del hecho de que a pesar de que compartimos la misma naturaleza humana, somos diferentes en muchas maneras. (p. 18)

En este mismo sentido, la formación de ciudadanía, según Ocampo, Méndez y Pavajeau (2008), implica cuidar del otro; acción que es denominada por ellos como un imperativo moral, que conlleva a cuidarse a sí mismo y con el otro; es decir, a tejer relaciones fundamentales de convivencia a través de prácticas de solidaridad, justicia, tolerancia y civismo.

Ospina, Carmona y Alvarado (2014), afirman que las múltiples subjetividades e ideas que van construyendo los niños sobre la paz, se dan a partir de las relaciones y los contextos en que se desenvuelven; siendo la familia, la comunidad y la escuela, los más importantes. Ello evidencia, que a pesar de que una de las posibilidades que tienen los niños, niñas y adolescentes, es la de percibirse como víctimas en medio de comunidades que vulneran sus derechos fundamentales; también, tienen la oportunidad de reconocerse como actores sociales en comunidades que gesten nuevas formas de construcción de paz. Y, es allí donde juega un papel muy importante la escuela, ya que siendo uno de los contextos más importantes, en que los niños construyen sus ideas, concepciones y subjetividades respecto a temas como la paz; ésta, puede desde una configuración adecuada como escenario de paz, brindar las herramientas y aprendizajes necesarios para que todo niño, niña 
y adolescente tenga las mismas oportunidades de vivir y convivir en armonía, a pesar de las diversas dificultades que pueden estar inmersas en las relaciones interpersonales (Ferreyra, 2014).

Existen diversas propuestas de formación, que desde la escuela plantean la posibilidad de que los estudiantes adquieran habilidades para interrelacionarse de manera asertiva con sus pares y demás miembros, de la comunidad y de sus propias familias. Uno de ellos, es el programa de mediación escolar por pares, al que hace referencia Chaux et al. (2004), en el cual todos los miembros de la comunidad educativa deben seguir rigurosamente un protocolo de atención ante las situaciones de conflicto presentadas dentro de la institución educativa, que permiten la negociación y manejo de conflictos a partir de la adquisición de conocimientos y habilidades en el reconocimiento de los posibles conflictos a presentarse en el contexto escolar.

En este proceso de mediación, es necesario lograr reconocer las emociones intervinientes en el conflicto, para alcanzar así su autocontrol; de esta manera, será posible también, identificar las diversas formas de resolver constructivamente los conflictos, reconociendo la comunicación asertiva como una estrategia de prevención y manejo de situaciones conflictivas, en los estudiantes. En este mismo sentido, Suárez (2008), afirma que "la comunicación entre las personas es el soporte de la interacción, por eso si ésta resulta distorsionada o bloqueada, la resolución del conflicto puede darse en forma violenta o pasiva" (p. 191).

Otra propuesta de formación, es el modelo pedagógico llamado SaludArte, desarrollado en algunas instituciones educativas en México; el cual, plantea como eje central la educación para la ciudadanía, con el propósito de proporcionar a los estudiantes algunas herramientas que, desde el contexto educativo, les permita "conocerse, aceptarse e interactuar con los demás en condiciones de respeto y diálogo" (García y Klein, 2014; p. 8).

En el texto sobre Convivencia escolar que desarrolla el Gobierno de Chile (s.f.), a través de su Ministerio de Educación, mencio- 
nan que teniendo en cuenta que en la escuela existen "integrantes de distinta jerarquía y, sobre todo, de distintas generaciones, hace de la institución escolar un espacio proclive al surgimiento de conflictos que alteran el buen clima que debe acompañar los procesos de aprendizaje que se desarrollan en su interior" (p. 1). Es decir que, partiendo de que la convivencia escolar es un proceso cotidiano entre los diferentes miembros de una comunidad educativa; el conflicto se configura como una forma de interacción entre dichos miembros, y consecuentemente, también en una forma de construcción, donde a pesar de afectar el clima escolar, favorece el proceso de enseñanza- aprendizaje, que debe ser permanente y responsable por parte de todos los miembros de la comunidad educativa. Además, afirman:

La convivencia consiste en gran medida en compartir, y a compartir se aprende. A compartir tiempos y espacios, logros y dificultades, proyectos y sueños. El aprendizaje de valores y habilidades sociales, así como las buenas prácticas de convivencia, son la base del futuro ciudadano. $Y$ ese aprendizaje tiene lugar en gran medida en la experiencia escolar. (Gobierno de Chile, s.f.; p. 2)

Como afirma Benítez (2009), en el contexto escolar uno de los mayores retos es lograr la coherencia entre lo que piensa, dice y hace el ser humano, transformando aquello que se encuentra oculto en él; partiendo de que "una escuela que apueste por la educación y la cultura de PAZ puede y debe lograr construir un nuevo conjunto de relaciones instaladas en los principios, valores y prácticas consecuentes con el espíritu de los $\mathrm{DDHH}^{\prime \prime}$ (p. 148).

En la escuela, actualmente, se puede observar en mayor medida, la concepción del conflicto "como un algo negativo que hay que anular, expulsar, negar o invisibilizar" (Benítez, 2009; p. 153). Sin embargo, ello denota una contravía frente a la construcción de paz en el contexto escolar, debido a que en ausencia de conflicto no sería necesaria siquiera, la educación o cultura de paz. En otras palabras, es importante reconocer que el conflicto posibilita la construcción y resignificación de la convivencia y la paz, par- 
tiendo de que la comunidad está integrada por seres humanos, y todos merecen ser tratados con dignidad.

En el marco del desarrollo humano, el reto que poseen hoy en día las instituciones educativas, es trabajar por la corresponsabilidad en la formación ciudadana de los estudiantes, tanto a nivel personal, como familiar e institucional; donde todas las partes, deben reconocer su responsabilidad y rol que ejercen en dicha formación de ciudadanía, de tal manera que ninguno suplante o sustituya la función del otro; $y$, donde el educando debe empoderarse de su propia formación. Es así que, "el conflicto y las confrontaciones pueden surgir, es un hecho que la educación debe formar para la convivencia y educar para el conflicto" (Touriñán, 2007; p. 269).

Ahora bien, para que la escuela sea efectivamente pensada y reconocida como un escenario de paz, y para que los actores intervinientes, especialmente los profesores como constructores de paz y de conocimiento, ejerzan adecuadamente su rol, hace falta que adquieran conocimientos en el tema y desarrollen ciertas habilidades, como, por ejemplo:

La escucha activa, atención, confianza en el otro, organización, producir, participar, crear estrategias para la paz, trabajo en equipo, memoria, dialogo y comunicación, toma de decisiones, demostrar afecto, comprender y reflexionar la realidad escolar, reconocer sus emociones en relación a la construcción de paz y resolución de conflictos. (Mora, 2016; p. 77)

Mora (2016), concluye que además de aspectos como la alegría, la entrega y el respeto, los docentes deben valorar el pluralismo, como una herramienta para reconocer al otro, sus capacidades y potencialidades, su rol e importancia para la construcción de una sociedad en paz; donde ésta sea aprendida y vivida, para potenciar la vida y la condición humana. Por ello, propone: "Si se hace de las prácticas diarias espacios de sana convivencia y justicia, los niños, niñas y jóvenes empezarán a percibir una realidad 
tranquila, que les genera ciertos referentes conceptuales, aptos para la promoción de conductas pacíficas" (p. 79).

Por último, es de resaltar que en su labor, los profesores tienen la obligación de brindar una formación integral a sus estudiantes, de tal manera que adquieran conciencia sobre los problemas y conflictos que se puedan presentar, tanto a nivel social como escolar, y así "desarrollar estrategias para darles solución, enseñar a solucionar el conflicto de forma no-violenta y desechar puntos de vista e ideas estereotipadas que pueden afectar el desarrollo humano" (Castillo \& Gamboa, 2012; p. 131).

Es importante resaltar la dimensión pedagógica y humana de la escuela, ya que en ella se desarrollan los procesos de socialización y construcción de sentidos de identidad, dados a través de la interacción y la confrontación con pares, maestros y demás personas inmersas en la comunidad educativa. Dichas interacciones deben darse en torno a las pautas o normas de convivencia previamente establecidas a nivel social o institucional, así como a los valores y principios que poseen los intervinientes; la responsabilidad y compromiso, el respeto y toma de decisiones, que favorecen la concepción de que "la escuela es un espacio de interacción, construcción y desarrollo de potencialidades necesarias para la comprensión del mundo, sus relaciones y sus posibles transformaciones" (Echavarría, 2003; p. 4).

\section{Método}

La investigación se adelantó a partir del enfoque Cualitativo con un alcance Descriptivo, partiendo de la postura metodológica propuesta por Hernández, Fernández y Baptista (2006), que permitió el análisis de la información recolectada a través de entrevistas semiestructuradas, con el objetivo de identificar y describir la manera como profesores, padres de familia y estudiantes, conciben la escuela como un escenario de paz y aquellas características que así la definen. 
Los participantes en este estudio, fueron veinticuatro (24) profesores, veinticuatro (24) padres de familia y veinticuatro (24) estudiantes, de grados octavo y noveno de básica secundaria, de tres instituciones educativas, dos de ellas ubicadas en las ciudades de Bogotá y Villavicencio, en Colombia; y, la tercera en la ciudad de Quito en el Ecuador.

La muestra fue seleccionada a través de un muestreo no probabilístico por conveniencia (Battaglia, 2008a; citado por: Hernández et al, 2006), conformada por un total de 72 participantes, a los cuales se tenía acceso y que cumplían los criterios de selección. Los participantes se encontraban distribuidos de la misma manera para cada una de las tres instituciones educativas, así: cuatro (4) estudiantes de grado octavo y cuatro (4) de grado noveno; dos (2) niños y dos (2) niñas, de cada grado. De igual manera, cuatro (4) padres de familia y cuatro (4) profesores, tanto de grado octavo, como de grado noveno.

Se construyó una encuesta semiestructurada, con 13 preguntas; la cual fue evaluada y validada por expertos. Por medio de dicha encuesta, se plantearon las mismas preguntas a toda la muestra participante en la investigación: profesores, padres de familia y estudiantes.

\section{Procedimiento}

La investigación fue adelantada en tres fases, así:

\section{Primera fase: Selección de la muestra; y, diseño y validación} del instrumento. Durante esta primera fase, se determinó como criterio principal de selección de la muestra poblacional, que fueran estudiantes de grados octavo o noveno, o que fueran docentes o padres de familia de los estudiantes, de dichos grados; seleccionados por conveniencia. Simultáneamente se diseñó y presentó para validación por expertos la entrevista semiestructu- 
rada, como instrumento de recolección de información sobre las concepciones que poseen los participantes.

Segunda fase: Aplicación de la entrevista semiestructurada. Para el desarrollo de esta fase, inicialmente, fueron diligenciados los respectivos consentimientos informados por parte de los participantes o sus acudientes (para el caso de los menores de edad). Luego, se procedió a iniciar las entrevistas, las cuales fueron grabadas y posteriormente transcritas, para facilitar el desarrollo de la siguiente fase.

Tercera fase: Codificación, Categorización y Análisis de la información. Esta fase, se adelantó a partir de la Teoría Fundamentada de Strauss \& Corbin (2002), quienes proponen un manejo sistemático de los datos recolectados, por lo cual se realizó inicialmente, procedimientos de codificación abierta y codificación axial; lo que permitió posteriormente, clasificar la información según algunas categorías de análisis emergentes de los datos recolectados y codificados; pero teniendo en cuenta también, aquellas propuestas desde la teoría. Y, es que "Debido a que las teorías fundamentadas se basan en los datos, es más posible que generen conocimientos, aumenten la comprensión y proporcionen una guía significativa para la acción" (Strauss \& Corbin, 2002; p. 22).

\section{Resultados}

A continuación, se presentan los resultados obtenidos durante la aplicación de entrevistas a los profesores, padres de familia y estudiantes de los grados octavo y noveno, pertenecientes a las tres instituciones educativas. De las respuestas dadas por dichos participantes, y teniendo como punto de partida los ejes conceptuales que surgieron durante la revisión bibliográfica, surgieron algunas categorías de análisis con sus respectivas subcategorías; las cuales se relacionan con las voces de los participantes, las 
ideas que ellos expresaron durante el desarrollo de la entrevista, que hacen referencia a cada subcategoría.

\section{Categoría 1. Paz:}

\section{Subcategoría 1.1. Ausencia de conflicto:}

En esta primera subcategoría los participantes expresaron que la Paz es ausencia de conflicto, así: "vivir todo en tranquilidad, en armonía, sin peleas, todos siendo amigos de todos"; "vivir sin conflictos"; "es que no haya guerras, ni conflictos, ni nada, que todo esté en armonía"; "se refiere a la ausencia de conflictos, guerras, discrepancia".

\section{Subcategoría 1.2. Convivencia armónica:}

En esta segunda subcategoría los participantes expresaron que la Paz se relaciona con la convivencia armónica, así: "primordialmente armonía, tranquilidad y convivencia sana, convivencia en armonía, fraternidad"; "un estado de sana convivencia donde coexisten diferentes individuos, hablemos de persona- persona, persona- medio ambiente, en sí, del individuo en su entorno"; "es la sana convivencia en una comunidad o sitio".

\section{Categoría 2. Familia:}

\section{Subcategoría 2.1. Orientación:}

En esta primera subcategoría los participantes expresaron que la familia tiene el deber de aportar a la paz orientando, así: "Los padres de familia deben ser personas muy comprometidas en la vigilancia, en la orientación"; "la paz se construye desde el hogar"; "Explicando a nuestros hijos de la importancia y el progreso que trae la paz y los beneficios para todos". 


\section{Subcategoría 2.2. Apoyo:}

En esta segunda subcategoría los participantes expresaron que la familia debe brindar apoyo en el proceso de construcción de paz, así: "el apoyo que le deben de brindar a los maestros dentro de esa enseñanza que ellos están impartiendo en las aulas"; "brindando apoyo a los directores, revisando las acciones de nuestros hijos cada día, estableciendo normas claras, apoyo institucional", "cualquier cosa mi mamá siempre está aquí o en las actividades de pastoral siempre me va a ver o trata de ayudar".

\section{Subcategoría 2.3. Formación:}

En esta tercera subcategoría los participantes expresaron que la familia debe cumplir una función formativa, y que de esta manera aporta al proceso de construcción de paz, así: "En la familia se debe enseñar valores, el respeto, tener respeto por las mujeres no discriminar"; "es desde la familia que empiezan todos los valores para llegar al colegio y saber estar en convivencia con todos los compañeros"; "la paz es un valor que hay que rescatar empezando por la familia que es el núcleo de la sociedad"; "que cuente con los valores y el temor de Dios, para enseñar el respeto y la tolerancia hacia los demás", "formación en valores, lo que te decía, la parte de escucha, la parte de diálogo, la parte de amor como tal es muy necesaria en la familia como primer formador de paz".

\section{Categoría 3. Escuela:}

\section{Subcategoría 3.1. Atención a padres:}

En esta primera subcategoría los participantes expresaron que la escuela abre espacios de atención y participación de los padres en la escuela, así: "Se hace actividades los sábados con tal que venga la familia; la institución también busca mecanismos, hay una hora de atención a padres de familia, se les atiende encantados de la vida"; "por medio de la escuela de padres, hacer talleres 
con los padres, y no solo con los padres, sino con los hijos de una vez, donde se den esos encuentros de diálogo y de pautas para lograr esos procesos de paz"; "educación respecto a la familia, escuela de padres"; "generar esa triada de padres de familia, estudiantes y docentes, porque uno aprende bastante dialogando, tratando con los padres de familia".

\section{Subcategoría 3.2. Formación para la ciudadanía:}

En esta segunda subcategoría los participantes expresaron que la escuela debe brindar formación en aquellos aspectos que se relacionan con la paz, como la ciudadanía, así: "formación en valores humanos y comunicación asertiva"; "estamos acompañados de profesores y tutores y nos ayudan para aprender a vivir entre compañeros, a escuchar al otro y respetar su manera de pensar"; "se enseñe la paz como si fuera un valor más en la vida del ser humano"; "Si alguien da una opinión sobre lo que él piensa y si alguien más se burla, el maestro incentivar a que todos tienen el mismo derecho a opinión"; "donde cada uno cumpla lo que le corresponde a cada uno responsablemente, que todos seamos coherentes con las normas, si hay respeto podemos tener una convivencia de paz. Que las normas estén dadas a conocer con anterioridad y se deberá en algún momento también dar sanciones".

\section{Subcategoría 3.3. Actividades pedagógicas, culturales y deportivas:}

En esta tercera subcategoría los participantes expresaron que la escuela puede aprovechar todos los espacios institucionales para formar y construir paz, así: "nos llevaron a un centro especial y a cada uno nos tocó cuidar a un niño, en diferentes salones con capacidades especiales, diferentes cada uno. Creo que todos aprendimos que no solo porque alguien tenga una discapacidad significa que debemos hacerlo a un lado sino que podemos convivir con ellos sin ningún problema"; "Cuando se abren los juegos, los Inter cursos, cada actividad que hay todos se reúnen, todos 
cooperan, a cada quien le toca hacer un trabajo y eso es trabajo en equipo, así que creería que también es parte de la paz"; "algunos estudiantes dan ideas y de ahí el profesor como que los guía, pone trabajos en equipos, así actividades y pues uno se alegra, coopera, ayuda, hace todo lo posible, de ahí viene la amistad y el respeto y todo", "lo que ayuda es que se integren a través de juegos", "oficina de bienestar estudiantil, actividades de inclusión y escuela de liderazgo", "como herramienta se desarrollan actividades lúdicas y culturales", "capacitaciones, actividades de retiros".

\section{Subcategoría 3.4. Constructores de paz:}

En esta cuarta subcategoría los participantes expresaron que los profesores deben ser constructores de paz por excelencia (Pérez, 2014), pero que para ello deben poseer algunas cualidades, de las cuales se mencionan algunas a continuación: "total paciencia, que sea sincero"; "el ejemplo de un profesor. No gritándolos, dialogando con ellos, explicándole las cosas como son"; "conocimiento ante todo, también una buena formación en valores"; "un principio de equidad, de justicia y pues de conocimiento, lo que te decía de conocimiento de sociedad, de las normas que maneja"; "que respete las creencias y las ideologías de las demás personas, en este caso de los estudiantes; debe ser muy tolerante, debe ser una persona que entienda a los estudiantes, de donde vienen, sus estados de ánimo"; "tener empatía con los estudiantes"; "que sepa dialogar, que sepa tomar las cosas con calma y transmitir esa sensación de tranquilidad, de calma y de diálogo a sus estudiantes"; "que le explique a uno bien, si uno no entiende pues que le vuelva a explicar, sin necesidad de regaños, sin necesidad de bullying"; "un líder y que sepa manejar esto con mucho equilibrio"; "capaz de transmitir directamente la paz, impartida a través de los conocimientos, que de un buen trato a los estudiantes, que sea capaz de impartir una buena educación y eso prácticamente contribuye"; "la espiritualidad, esa es una característica"; "estar comprometido con la misión y visión de la educación"; "no es problemático"; "Consciente de la importancia de ser formadores de paz"; "Poniendo en práctica lo que enseña y sobre todo ser 
tolerante"; "ser proactivo en la enseñanza de la paz"; "capacidad de escuchar, ser asertivo en la comunicación"; "siendo ejemplo de respeto, de conciliación, de diálogo y de tolerancia con sus estudiantes".

\section{Categoría 4. Convivencia:}

\section{Subcategoría 4.1. Diálogo:}

En esta primera subcategoría los participantes expresaron que el diálogo es un componente importante en la convivencia, así: "Siempre considero yo importante la conciliación por medio del diálogo, el diálogo no solo entre docentes y estudiantes, y entre estudiantes, sino también con nosotros que somos parte de esta trilogía de la educación"; "siempre hablamos, nunca a los golpes cuando hay problemas, así, solo hablando, nos sentamos los conversamos y lo resolvemos"; "El diálogo, pedir disculpas y buscar nuevos acuerdos para que no se repita el problema o discusión"; "Ser capaces de resolver un problema con el diálogo"; " construcción de paz mediante el diálogo"; "es importante el diálogo y la escucha".

\section{Subcategoría 4.2. Resolución de conflictos:}

En esta segunda subcategoría los participantes expresaron que la resolución de conflictos debe ser parte de la convivencia, así: "enseñarles desde las aulas a tener una resolución de conflictos mediante el diálogo"; "cuando hay problemas no debería haber gritos, deberían dialogar de una manera tranquila y ver qué es lo que pasa y cuáles son las soluciones"; "cuando tenga algún problema algún estudiante, para que no se vaya directamente a suspensión o expulsión, sino que se busque una solución de porqué paso aquel incidente y saber porque lo hicieron".

\section{Subcategoría 4.3. Respeto:}

En esta tercera subcategoría los participantes expresaron que el respeto debe ser complemento de la convivencia, así: "debe- 
ría poder aceptar y respetar las opiniones del resto"; "El respeto forma parte para poder formar un ambiente de paz"; "enseñar el respeto y la tolerancia hacia los demás"; "buena convivencia con sus compañeros con mucho respeto"; "respeto por la vida y mejores relaciones entre las personas"; "enseñarles a convivir con los otros, dándoles el respeto que cada uno se merece, crearles consciencia sobre el temor a Dios"; "el respeto a la palabra de cada uno de los integrantes de la familia".

\section{Categoría 5. Transformación personal:}

\section{Subcategoría 5.1. Resiliencia:}

En esta primera subcategoría los participantes expresaron que la resiliencia es importante en el proceso de transformación personal para alcanzar la paz, así: "en todas partes hay dificultades pero son superables"; "yo siento que el colegio al menos para mí, me ha ayudado bastante, superando primero una depresión"; "superar de una vez por todas aquellas condiciones que afectan la convivencia de ellos mismos dentro del establecimiento educativo"; "asumen su responsabilidad y cambian positivamente"; "debe estar básicamente como persona muy abierta a los cambios"; "cambiando su actitud ante los problemas"; "disponibles siempre a cambiar y ser mejores en la sociedad"; "dedicación y compromiso al cambio".

\section{Categoría 6. Cambio social:}

\section{Subcategoría 6.1. Apoyo interinstitucional:}

En esta primera subcategoría los participantes expresaron que un cambio social requiere el apoyo de diversas entidades, a las instituciones educativas, así: "buscar convenios de otras entidades que aporten experiencia y motivación a los estudiantes"; "Tener convenio de otras entidades para el aporte social"; "recibimos apoyo de entidades externas del gobierno, la policía y tantas 
charlas que son importantísimas para nuestros jóvenes"; "vienen incluso otras instituciones como el patronato San José estuvo impartiendo unas campañas contra el bullying, estuvo presente también el DINAPEN, son instituciones que vienen".

\section{Discusión}

El objetivo principal de esta investigación fue conocer y describir las concepciones que tienen los padres de familia, profesores y estudiantes de grados octavo y noveno de básica secundaria, sobre la escuela como escenario de paz, partiendo del reconocimiento del rol de la familia y la escuela en este proceso, así como de los recursos que posee la escuela para su consolidación como escenario de paz. Ahora bien, dentro de los resultados se encontró que la información obtenida en las tres instituciones educativas, es homologable entre ellas, debido a que los aspectos que se tuvieron en cuenta son comunes en las tres; es decir, que no se observaron diferencias marcadas en las concepciones de la escuela como escenario de paz.

Es así que es posible afirmar, que los participantes conciben que un escenario de paz puede darse de dos grandes maneras, la primera de ellas en ausencia de conflicto, cuando no hay peleas, guerras o conflictos (Benítez, 2009); y la otra, cuando se logra una convivencia en armonía, cuando hay fraternidad y un ambiente sano para convivir con el otro. Esta última concepción, afín a la definición de convivencia y paz dada por Chaux, Lleras y Velásquez (2004), fue la más frecuente entre los participantes, más aún cuando se fue profundizando en aquellos aspectos y recursos con que debe contar las escuelas.

Por otro lado, al hacer referencia al rol de la familia, se encontró que ésta es concebida como la principal institución de formación en aquellos aspectos que componen los procesos de paz, como lo son los valores y principios como el respeto, la tolerancia, el amor, lo cual deben hacer utilizando como estrategia la orientación y apoyo entre miembros de la familia, especialmente por medio del diálo- 
go de los padres a los hijos, así como siendo modelos a seguir a través del ejemplo. De igual manera, es importante que la familia se empodere de las diferentes alternativas de acompañamiento del proceso de sus hijos en las instituciones educativas, no solo desde casa brindando apoyo en los aspectos netamente académicos, sino también, brindando apoyo a los demás miembros de la comunidad educativa y ejerciendo su rol de participación y representación, lo cual se relaciona directamente con los planteamientos que al respecto hizo Touriñán (2007).

En cuanto al rol que debe ejercer la escuela, se encontraron cuatro grandes aspectos a tener en cuenta. El primero de ellos, es que los participantes consideran que es de gran importancia que exista un espacio de comunicación permanente entre los padres de familia y las instituciones educativas, de tal manera que sea posible atender las necesidades y requerimientos de participación, formación e información, bidireccionalmente; de hecho, en las tres instituciones educativas se hizo mención de la importancia que tiene la escuela de padres, como espacio estratégico para alcanzar este objetivo.

Así mismo, se encontró que se le asigna a la escuela la responsabilidad de la formación de sus estudiantes, para la ciudadanía; es decir, que a pesar de que los valores y principios son concebidos como responsabilidad de la familia, su aplicabilidad y ejercicio permanente como parte de la interacción social del ser humano, se aprende y propicia a partir de la formación en valores y derechos humanos, así como fortaleciendo habilidades como el diálogo, la comunicación asertiva, la resolución de conflictos, la escucha activa, el respeto mutuo. Y, es que la formación y desarrollo de competencias para la convivencia, como estrategia de prevención de la violencia desde las escuelas, ha ido tomando cada vez más fuerza por diversos autores (Chaux et al., 2004; Chaux y Velásquez, 2008; Suárez, 2008; Tibbitts, 2002; Ocampo, Méndez y Pavajeau, 2008; Echavarría, 2003).

Los participantes expresan que el aprovechamiento de las actividades académicas y de las extracurriculares (Konieczny, 2015), puede funcionar como una gran estrategia de construcción de 
paz y desarrollo de habilidades en los estudiantes; ello, debido a que dichas actividades exponen a los diferentes miembros de la comunidad educativa, a diversas situaciones en las cuales deberán hacer uso de habilidades adquiridas para relacionarse de manera armónica con los otros, o en caso contrario, para resolver o mediar cualquier situación de conflicto que pueda presentarse, teniendo en cuenta que éstos son coexistentes a la interacción de los seres humanos y constituyen una forma de relacionarse con los demás y de adquirir nuevos conocimientos sobre la convivencia(Suárez, 2008; Alzate, 2007; Chaux et al., 2004).

Los participantes conciben a los profesores como "Constructores de paz" por excelencia, y mencionan que para ello deben poseer cualidades como: la paciencia, sinceridad, empatía, tranquilidad, capacidad de diálogo y resolución de conflicto, valores y principios como el respeto y la tolerancia, así como cumplir las normas de convivencia, liderazgo positivo, compromiso con la educación, espiritualidad, coherencia entre lo que enseña y hace, capacidad de escucha, asertividad en la comunicación y habilidades de resolución y mediación de conflictos; cualidades que han sido mencionadas por Mora (2016).

Por otro lado, fue posible observar que algunas de las cualidades y habilidades que se espera deben poseer los profesores que construyen paz en las instituciones educativas, son concebidas como grandes pilares de la convivencia armónica, por lo cual deben ser desarrolladas por los diferentes miembros de la comunidad educativa; entre ellas, el diálogo, la resolución de conflictos y el respeto, los cuales constituyen según García y Klein (2014), herramientas para conocerse, aceptarse e interactuar con los demás, como parte del proceso de convivencia. De dichas habilidades, el diálogo es el más frecuente, tanto en casa a nivel familiar, como en la escuela con los diferentes miembros de la comunidad educativa, y se encuentra relacionado directamente con la escucha activa. También está la resolución de conflictos, ya que como fue mencionado anteriormente, la escuela como escenario de paz es pensada por la mayoría de los participantes como un escenario en el cual la paz no hace referencia a la ausencia de conflicto, sino 
a la habilidad o capacidad de resolverlos. El respeto, también es considerado por los participantes, como de gran importancia para lograr construir paz en las instituciones educativas, y afirman que consiste en la tolerancia y aceptación del otro como diferente en la medida en que cada ser humano es único e irrepetible, pero a la vez, igual en términos de derechos humanos.

Por último, los participantes expresaron que, para poder alcanzar la transformación de las escuelas como escenarios de paz, se debía partir de una transformación personal, para lo cual resulta necesario poseer capacidad de resiliencia, en la medida en que en algunas ocasiones el cambio implica ensayo y error, pero el éxito depende en gran medida de esa capacidad de superación y constancia, de mantener la mirada siempre en el objetivo a alcanzar. Así mismo, debe haber un cambio a nivel social, especialmente de aquellas instituciones $u$ organizaciones externas o gubernamentales, que pueden y deben aportar en la construcción de las escuelas como escenario de paz; es decir, que todos los agentes inmersos en los diferentes niveles del sistema educativo, deben brindar apoyo interinstitucional en dicho proceso de construcción de la escuela como escenario de paz (Reboul, 1972; Tedesco, 1995; Morín, 2000; Touriñán, 2004a; citados por: Touriñán, 2007).

\section{Referencias bibliográficas}

Alzate, R. (2007). Teoría del conflicto. Madrid: Universidad Complutense de Madrid. Recuperado de: https://mediacionesjusticia.files.wordpress. com/2013/04/alzate-el-conflicto-universidad-complutense.pdf

Benítez, L. (2009). Educación y construcción de culturas de paz en la escuela: de la práctica a la teoría. Pensamiento Jurídico, (26), 143- 160. Recuperado de: https://revistas.unal.edu.co/index.php/peju/article/ view/36561/38480

Castillo M, \& Gamboa, R. (2012). La educación para la paz: una respuesta a las demandas sociales. Rev. Electrónica Diálogos Educativos 
12 (23), 117- 133. Recuperado de: http://revistas.umce.cl/index.php/ dialogoseducativos/article/view/1064/1076

Chaux, E., Lleras, J., \& Velásquez, A. (2004). Competencias ciudadanas: de los estándares al aula. Una propuesta de integración a las áreas académicas. Bogotá: Ediciones Uniandes. Recuperado de: https:// www.colombiaaprende.edu.co/html/mediateca/1607/articles-75077_ archivo.pdf

Chaux, E., \& Velásquez, A. (2008). Violencia en los colegios de Bogotá: contraste internacional y lagunas recomendaciones. Revista Colombiana de Educación: Investigaciones (55), 14- 37. Recuperado de: http:// revistas.pedagogica.edu.co/index.php/RCE/article/view/7569/6085

Constitución de la República del Ecuador (2015). Recuperado de: http:// www.asambleanacional.gov.ec/documentos/constitucion_de_bolsillo. pdf

Constitución Política de Colombia [Const.] (1991). Recuperado de: http:// www.corteconstitucional.gov.co/inicio/Constitucion\%20politica\%20 de\%20Colombia.pdf

de la Calle, C. V., Malaver, M. O., Gallego, J. D. M., Rodríguez, M., Flórez, J. C., Henao, C. E. \& Saldaña, R. (2014). Aportes de los doctorados de educación en ciencia, tecnología y sociedad, desde la sistematización de sus investigaciones doctorales científicas y formativas, 2000-2010. Revista Interamericana de Investigación, Educación y Pedagogía, RIIEP, 7(1). DOI: https://doi.org/10.15332/s1657-107X.2014.0001.04

Echavarría, C. (2003). La escuela: un escenario de formación y socialización para la construcción de identidad moral. Revista Latinoamericana de Ciencias Sociales, Niñez y Juventud, 1(2), 15-43. Recuperado de: http://www.scielo.org.co/scielo.php?pid=S1692715X2003000200006\&script=sci_abstract \&tlng=es

Ferreyra, H. A. (2014). Mesas Socioeducativas para la Inclusión y la Igualdad. Un programa "De todos con todos". Una experiencia en construcción. Revista Interamericana de Investigación, Educación y Pedagogía, RIIEP, 7(2). DOI: https://doi.org/10.15332/s1657107X.2014.0002.01 
Fisas, V. (1998). Cultura de Paz y Gestión de Conflictos. Capítulo XI: Una cultura de paz. Icaria/NESCO: Barcelona. Recuperado de: http:// escolapau.uab.cat/img/programas/cultura/una_cpaz.pdf

Fondo de las Naciones Unidas para la Infancia [UNICEF] \& Facultad Latinoamericana de Ciencias Sociales [FLACSO] (2011). Clima, Conflictos y Violencia en la Escuela. Argentina: Recuperado de: https://www. unicef.org/argentina/spanish/clima_conflicto_violencia_escuelas.pdf

García, B. Klein, I. (2014) La construcción de ambientes educativos para la convivencia pacífica: el modelo pedagógico del programa SaludARTE. Recuperado el 05 de diciembre del 2016, de http://www.scielo.org.mx/ scielo.php?script=sci_arttext\&pid=S1665-109X2014000100006

Gobierno de Chile. Ministerio de Educación (s.f.). Convivencia escolar y resolución de conflictos: Material de apoyo para profesoras y profesores. Nuestros temas, (30). Recuperado de: http://portales.mineduc.cl/ usuarios/basica/File/Separata30.pdf.

Hernández, R., Fernández, C. y Baptista, P. (2006). Metodología de la investigación. México: Mc Graw Hill.

Konieczny, P. (2015). Lorenzo García Aretio: bases, mediaciones y futuro de la educación a distancia en la sociedad digital. Revista Interamericana de Investigación, Educación y Pedagogía, RIIEP, 8(1). DOI: https://doi. org/10.15332/s1657-107X.2015.0001.08

Langer, E. (2016). La construcción de confianza para el estudio de prácticas de resistencia en la escolarización de jóvenes en contextos de pobreza urbana. Revista Interamericana de Investigación, Educación y Pedagogía, RIIEP, 9(2). DOI: https://doi.org/10.22490/25391887.1945

Ministerio de Educación del Ecuador [MEE], Fondo para las Naciones Unidas para la Infancia [UNICEF] \& World Vision (2015). Una mirada en profundidad al acoso escolar en el Ecuador. Violencia entre pares en el sistema educativo. Recuperado de: https://educacion.gob.ec/wpcontent/uploads/downloads/2017/05/Una_mirada_en_profundidad_ al_acoso_escolar_en_el_Ecuador.pdf 
Mora, P. (2016). Conocimientos, habilidades y actitudes de los docentes constructores de paz. Mundo Fesc, (11), 72- 79. Recuperado de: http://www.fesc.edu.co/Revistas/OJS/index.php/mundofesc/article/ view/84/133

Ocampo, A., Méndez, S. \& Pavajeau, C. (2008). Las subjetividades como centro de la formación ciudadana. Universitas Psychologica, 7 (3), 837851. Recuperado de: http://www.scielo.org.co/pdf/rups/v7n3/v7n3a17. pdf

Ospina, M., Carmona, J. \& Alvarado, S. (2014). Niños en contexto de conflicto armado: Narrativas generativas de paz. Revista Infancias Imágenes, 13 (1), 52- 60. Recuperado de: http://ceanj.cinde.org.co/ programa/Archivos/publicaciones/p1/_1_AN_255.pdf

Pérez, T. H. P. (2013). Aproximaciones al estado de la cuestión de la investigación en educación y derechos humanos. Revista Interamericana de Investigación, Educación y Pedagogía, RIIEP, 6(1). DOI: https://doi. org/10.15332/s1657-107X.2013.0001.05

Pérez, T. H. (2014). Colombia: de la educación en emergencia hacia una educación para el posconflicto y la paz. Revista Interamericana de Investigación, Educación y Pedagogía, RIIEP, 7(2). DOI: https://doi. org/10.15332/s1657-107X.2014.0002.06

Strauss, A. \& Corbin, J. (2002) Bases de la Investigación Cualitativa. Técnicas y Procedimientos para Desarrollar la Teoría Fundamentada. Bogotá: Contus - Editorial Universidad de Antioquia. Recuperado de: $\quad$ https://diversidadlocal.files.wordpress.com/2012/09/basesinvestigacion-cualitativa.pdf

Suárez, O. (2008). La mediación y la visión positiva del conflicto en el aula, marco para una pedagogía de la convivencia. Revista DiversitasPerspectivas en Psicología, 4 (1), 187- 199. Recuperado de: http:// revistas.usta.edu.co/index.php/diversitas/article/view/275/465

Tahull, J. (2016). Modernidad, educación y género. El proyecto inacabado. Revista Interamericana de Investigación, Educación y Pedagogía, RIIEP, 9(2), 159-178. DOI: https://doi.org/10.22490/25391887.1947 
Tibbitts, F. (2002). Understanding what we do: emerging models for human rights education. International Review of Education, 48 (3-4), 159-171. DOI: 10.1023/A:1020338300881.

Touriñán, J. (2007) Valores y convivencia ciudadana: Una responsabilidad de formación compartida y derivada. Bordón. Revista de Pedagogía, 59 (2-3), 261-312. Recuperado de: https://dialnet.unirioja.es/descarga/ articulo/2552443.pdf 